\title{
L'ATTUALITÀ DELLE SCELTE ISTITUZIONALI, FINANZIARIE E DI POLITICHE DELLE COMUNITÀ EUROPEE PRIMA DEI TRATTATI DI ROMA
}

\author{
JACQUES ZILLER (*) \\ Nota presentata dal m.e. Silvio Beretta \\ (Adunanza del 28 novembre 2019)
}

SunTo. - I trattati di Parigi che istituivano la CECA e la CED, nonché il progetto di Statuto della Comunità politica europea, pur anteriori ai trattati di Roma e non ormai in vigore, costituiscono la fonte più importante dell'assetto istituzionale dell'attuale Unione europea e meritano quindi di essere studiati di nuovo, anche nella prospettiva di ulteriori riforme dell'UE. Lo stesso vale per il diritto derivato e le politiche che ne derivano, anzitutto per la politica industriale, nonché per quanto riguarda protezione dei lavoratori e dell'ambiente inclusi nel trattato CECA. Una particolare attenzione in questo senso è da dedicare alle Memorie di Jean Monnet.

$$
* * *
$$

RÉsumé. - Les traités de Paris instituant la CECA et la CED, ainsi que le projet de statut de la Communauté politique européenne, qui sont antérieurs aux traités de Rome et ne sont pas actuellement en vigueur, constituent la source la plus importante du dispositif institutionnel de l'Union européenne actuelle et méritent donc d'être étudiés à nouveau, y compris en vue de la poursuite de la réforme de l'UE. Il en va de même pour le droit dérivé et les politiques qui en sont issues, principalement la politique industrielle, et la protection des travailleurs et de l'environnement incluses dans le traité CECA. À cet égard, il convient d'accorder une attention particulière aux Mémoires de Jean Monnet.

(*) Professore ordinario di diritto dell'Unione europea all'Università di Pavia; Dipartimento di Scienze Politiche e Sociali, Pavia, Italy. E-mail: Jacques.ziller@unipv.it 


\section{ORIGINI DELL'INDAgINE SUlLE COMUNiTÀ EUROPEE ANTERIORI AI TRATTATI DI ROMA DEL 1957}

Le origini di questa Nota sono di natura aneddotica nonché scientifica. Il 25 marzo 2017 sono stati celebrati in pompa magna i sessant'anni anni dell'Unione europea. Per uno studioso del diritto comunitario, ora dell'Unione europea, per di più nato a Parigi nel 1951 era ovvia l'inesattezza di tale anniversario. Dal punto di vista strettamente formale si dovrebbe sostenere che l'Unione europea è nata a Maastricht il 7 febbraio 1992 - se si ritiene che la firma di un trattato multilaterale possa essere considerata equivalente alla nascita dell'organizzazione internazionale da esso istituita - oppure il primo novembre 1993, cioè alla data dell'entrata in vigore del trattato di Maastricht. Dal punto di vista sostanziale, invece, non c'è dubbio che l'odierna Unione europea non sia la continuazione delle Comunità europee. La prima delle tre Comunità è nata con il trattato di Parigi che istituiva la Comunità europea del carbone e dell'acciaio (CECA) firmato, per l'appunto, il 18 aprile 1951 ed entrato in vigore il 23 luglio 1952. E questa la ragione per la quale l'Università di Pavia ha celebrato nel novembre 2017 l'anniversario dell'Unione europea con un convegno dedicato non ai Trattati di Roma - come è stato fatto in tante altre istituzioni - ma ben piuttosto all'Integrazione prima dei Trattati di Roma. ${ }^{1}$ Come sottolineato dalla curatrice degli atti del Convegno prof. Giulia Rossolillo: ${ }^{2}$

In un'epoca nella quale i valori posti alla base del processo di integrazione sembrano essere messi da molti fortemente in discussione e prevale l'esigenza di soluzioni tecniche di breve periodo sulla prospettiva di una riforma in senso politico dell'Unione che sappia rendere $\mathrm{i}$ cittadini europei protagonisti del loro destino, i trattati istitutivi della CECA, della CED e della CPE assumono dunque una particolare rilevanza. Se la CECA, con la sua struttura fortemente sovranazionale imperniata sul ruolo dell'Alta Autorità e il suo sistema di finanziamento

Gli atti del convegno sono stati pubblicati in G. Rossolillo (a cura di), L'integrazione europea prima dei Trattati di Roma, - Università di Pavia, Dipartimento di scienze politiche e sociali, 10 novembre 2017, Quaderni della Rivista "Il Politico", n. 64, Soveria Mannelli, Rubettino, 2019, 119 p.

2 G. Rossolillo, Prefazione, in G. Rossolillo (a cura di), L'integrazione europea prima dei Trattati di Roma, [v. Nota 1], 7-8. 
fondato su prelievi sulla produzione di carbone e acciaio, fornisce infatti l'esempio di un'organizzazione con un forte grado di indipendenza dai suoi Stati membri e di un bilancio finanziato con reali risorse proprie, la Comunità Europea di Difesa e la Comunità Politica Europea rappresentano la manifestazione dell'evidente e necessario legame esistente tra difesa e unione politica, e dunque dell'impossibilità di far vita a un vero esercito europeo in assenza di una politica estera unica e di un governo democratico sovranazionale.

In altre parole: nel momento in cui l'integrazione europea è messa in discussione anche negli Stati fondatori, l'idea era di indagare maggiormente, o di nuovo, ciò che è stato fatto negli anni Cinquanta potrebbe forse permettere di trovare qualche elemento cui ispirarsi sul cammino verso un'integrazione più ampia che molti vorrebbero percorrere ma che non tutti sono in grado di percorrere bene.

L'approccio è stato quello di studiare - o studiare di nuovo anzitutto i Trattati: quello di Parigi che aveva istituito la CECA ma anche i Trattati sulla Comunità europea di difesa (CED) firmato anch'esso a Parigi il 27 maggio 1952 - cioè due mesi prima dell'entrata in vigore del trattato CECA nonché il progetto di statuto sulla Comunità politica europea adottato il 10 marzo 1953 a Strasburgo dall'Assemblea ad hoc creata con questo scopo dai Ministri degli esteri dei sei Stati fondatori il 10 settembre 1952 - cioè due mesi dopo l'entrata in vigore del trattato CECA. L'Assemblea ad hoc era composta dai 78 delegati dell'Assemblea comune della CECA, integrata da 8 membri cooptati ( 3 per l'Italia, 3 per la Francia, 2 per la Germania), più 13 osservatori del Consiglio d'Europa, che non rappresentavano i paesi membri della CECA. Si ricorda che non era chiaro fino a poco tempo prima della firma del trattato CECA se il Regno Unito avrebbe o meno aderito. ${ }^{3}$ Per quanto riguarda il trattato CECA, entrato in vigore nel 1952 e rimasto in vigore per i cinquant'anni da esso previsti, cioè fino al 22 luglio 2002, si deve anche prendere in considerazione la prassi istituzionale basata sul Trattato, che può ispirare ancora oggi qualche progetto d'integrazione ulteriore nell'ambito economico e non solo.

L'approccio intellettuale seguito per questa indagine è quello fun-

3 L'A. rimanda a questo proposito alle sue considerazioni al momento della firma del Trattato di Lisbona: J. Ziller, Il Nuovo Trattato Europeo, Bologna, Il Mulino, 2007. 
zionalista. Seguendo tale approccio è utile definire anzitutto cosa fa e/o a cosa serve un'istituzione. Ed è anche l'unico modo per comprendere se un'istituzione è oggi è ancora adatta. Esaminando quindi le tre comunità progettate all'inizio degli anni Cinquanta del secolo scorso, è da notare che se la CECA, che ha funzionato fino al 2002 in quanto trattato di base per il settore siderurgico, gli altri due trattati, pur non essendo entrati in vigore, hanno col passare del tempo avuto degli "eredi". Ad esempio, la sostanza del trattato CED viene trattato nell'odierna UE con le disposizioni relative alla Politica di sicurezza comune, di cui si può sperare da un paio di anni che possa proseguire nella direzione di andare oltre qualche operazione umanitaria o qualche dichiarazione. Per quanto riguarda lo Statuo della comunità politica, una serie dei suoi elementi sono stati ripresi nel progetto di Trattato d'Unione europea del 1984 - di cui Altiero Spinelli è stato relatore al Parlamento europeo- a poco a poco ripresi nei trattati posteriori, anzitutto quelli di Maastricht e Lisbona.

\section{LE BASI DELL'ASSETTO GIURIDICO-ISTITUZIONALE DELL'UNIONE EUROPEA $^{4}$}

\subsection{La Dichiarazione Schuman e il metodo comunitario}

L'Unione europea odierna e senza dubbio l'erede delle Comunità europee, sia dal punto di visto politico, sia dal punto di vista giuridico, in quanto messa in opera della dichiarazione Schuman del 9 maggio 1950. Tale dichiarazione era stata il frutto di un intenso negoziato tra i consiglieri dei capi di governo dei 6 Stati fondatori, nonché del governo britannico, che tuttavia rinunciò qualche giorno prima a partecipare ad un progetto che includeva una nuova struttura sovranazionale. Per chi

\footnotetext{
${ }^{4}$ Vengono ripresi in questa sezione i principali argomenti sviluppati in J. Ziller, Le basi dell'assetto giuridico-istituzionale dell'unione europea nel trattato CECA e nei progetti CPE e CED, in G. Rossolillo (a cura di), L'integrazione europea prima dei Trattati di Roma, [v. Nota 1], 9-28. La sezione 4 invece è una sintesi degli altri lavori, in particolare quelli di Andrea Zatti sulle finanze della CECA e di Giulia Avanzini sulle origini della tutela dei lavoratori e della tutela dell'ambiente.

5 V. https://europa.eu/european-union/about-eu/symbols/europe-day/schuman-declaration_it.
} 
sostiene oggi che il progetto di comunità del carbone e dell'acciaio non riguardava problemi di sovranità, a differenza delle problematiche odierne della moneta unica e del controllo delle frontiere, basta ricordare, per l'appunto, la decisione britannica di rinunciare ad un progetto che limitava la sua sovranità, e il fatto che il controllo della produzione e vendita del carbone e l'acciaio era centrale per le potenze europee, all'origine, per l'appunto, delle due guerre mondiali del Novecento.

Due citazioni delle Memorie di Jean Monnet ${ }^{6}$ riguardo al contesto nel quale nacque il progetto della CECA dimostrano molto bene l'importanza politica del carbone e dell'acciaio all'inizio degli anni Cinquanta del secolo scorso. Monnet fa riferimento ad un suo documento, scritto in quanto Commissario al Piano:

La Francia cesserà la sua ripresa se non si regolerà rapidamente la questione della produzione industriale tedesca e della sua capacità di concorrenza. Il fondamento della superiorità che gli industriali francesi riconoscono tradizionalmente alla Germania è la produzione dell'acciaio a un prezzo con cui la Francia non può entrare in concorrenza. $\mathrm{Ne}$ concludono che tutta la produzione francese ne è handicappata. Già la Germania chiede di aumentare la sua produzione da undici a quattordici milioni di tonnellate. Noi rifiuteremo, ma gli americani insisteranno. Alla fine, faremo delle riserve, ma cederemo. Contemporaneamente, la produzione francese rimane stazionaria e perfino cala. ${ }^{\top}$

A differenza della maggior parte della classe politica francese, che voleva mantenere e rinforzare il controllo operato dall'Autorità internazionale della Ruhr - creata il 28 aprile 1948 da un accordo firmato da Belgio, Francia, Lussemburgo, Paesi Bassi, Regno Unito e Stati Uniti d'America,$-{ }^{8}$ il Ministro degli Esteri francese Robert Schuman aveva un atteggiamento molto più aperto verso il Cancelliere tedesco Konrad Adenauer e la classe politica democratica della Germania del dopoguerra. Fu quindi a Schuman che Monnet indirizzò le sue proposte, nate dall'idea così da lui descritta:

\footnotetext{
J. Monnet, Mémoires, Paris, Fayard, 1976; traduzione italiana J. Monnet, Cittadino d'Europa, con prefazione di Giorgio Napolitano, Napoli, Guida. 2007.

7 J. Monnet, Cittadino d'Europa [v. Nota 6], 261.

8 V. M. Deteix, L'Autorité internationale de la Rubr, Thèse, Parigi, 1951.
} 
l'azione avrebbe dovuto essere diretta sul punto dove il malinteso era più tangibile, dove si sarebbero di nuovo prodotti gli errori del passato. Se in Francia avessimo potuto cancellare il timore del predominio industriale tedesco, il più grande ostacolo all'unione dell'Europa sarebbe stato eliminato. Una soluzione che ponesse l'industria francese sulla stessa base di partenza dell'industria tedesca, pur liberando quest'ultima dalle discriminazioni derivate dalla disfatta, avrebbe potuto ristabilire le condizioni economiche e politiche per un'intesa indispensabile all'Europa. Anzi, avrebbe potuto essere il fermento stesso dell'unità europea. ${ }^{9}$

È anche utile ricordare che se il progetto di CED incontrò la resistenza di una maggioranza di fortuna all'Assemblée nationale francese nel 1954, mentre vi era stato una maggioranza per la ratifica del trattato CECA nella stessa assemblea, eletta il 17 giugno 1951, ciò non è tanto dovuto alla materia di cui si trattava, quanto all'evoluzione politica in un'epoca in cui l'instabilità di governo della $4^{\circ}$ Repubblica portava a compromessi dettati dall'attualità immediata e soprattutto dai giochi di partiti, e quindi di poca lungimiranza. L'assetto istituzionale dell'Unione è nato, per l'appunto, con la Dichiarazione Schuman e il trattato CECA.

In prima battuta occorre ricordare che il cd. metodo comunitario $^{10}$ fu ideato dal cerchio degli specialisti riuniti attorno a Jean Monnet, ideatore e primo dirigente capo del Commissariat du plan istituito nel 1946 per coordinare gli sforzi di ricostruzione dell'economia e delle infrastrutture della Francia duramente colpite dalla guerra e dall'occupazione tedesca. Cercando una soluzione per migliorare i rapporti tra Francia e Germania, Monnet la suggerì a Schuman, che cercava idee nuove in vista dell'incontro previsto per il 10 maggio 1950 con i Ministri degli Esteri americano Dean Acheson e britannico Ernest Bevin. Con i suoi collaboratori, Monnet redasse quindi un testo che Schuman comunicò a Adenauer l'8 maggio e poi, avendo avuto la notizia del suo benestare, lo sottopose all'approvazione del Consiglio dei Ministri francese. La stampa fu convocata nel pomeriggio per ascoltare la «dichiarazione Schuman» del 9 maggio 1950 (quindi cinque anni ed

9 J. Monnet, Cittadino d'Europa [v. Nota 6], 262.

10 V. tra tanti altri, L. Levi, G. Montani, F. Rossolillo, Tre introduzioni al federalismo, Napoli, Guida, 2005. 
un giorno dopo la capitolazione della Germania nazista), con cui il Governo francese faceva una proposta:

... mettendo in comune le produzioni di base e istituendo una nuova Alta autorità, le cui decisioni saranno vincolanti per la Francia, la Germania e i paesi che vi aderiranno, costituirà il primo nucleo concreto di una Federazione europea indispensabile al mantenimento della pace.

Il secondo paragrafo della Dichiarazione del 9 maggio è stato citato non a caso dal Presidente francese Macron nel suo discorso della Sorbona del 26 settembre 2017, nel quale ha esposto il programma della Francia per contribuire allo sviluppo dell'Unione europea: «L'Europa non è stata fatta: abbiamo avuto la guerra».

Come rammentato da Monnet, un ruolo fondamentale fu quello di Paul Reuter, giovane professore ordinario di diritto pubblico che era stato uno dei pochi della sua generazione ad interessarsi al diritto della concorrenza, cioè al diritto antitrust degli Stati Uniti d'America. ${ }^{11}$ Scrisse Monnet:

Il caso fece capitare nel mio ufficio, rue de Martignac, un giovane professore di diritto che non conoscevo e che ci serviva da consulente, credo, sulla legislazione antitrust che mi sembrava necessario rinforzare in Francia. Paul Reuter era un uomo della Francia orientale, calmo e con i piedi per terra, la cui brillante dialettica affrontava i problemi concreti del diritto e della politica, collocandoli al loro giusto posto. Insegnava teoria a Aix e veniva a Parigi a risolvere le difficoltà del Quai d'Orsay, di cui era uno dei tre giureconsulti. Mi accorsi subito che i rapporti tra Francia e Germania costituivano per lui una preoccupazione personale e professionale assieme: il diritto internazionale avrebbe potuto eliminare i conflitti di cui gli abitanti delle zone di confine erano le vittime costanti? ${ }^{12}$

Così Reuter fu coinvolto sin dall'inizio nella stesura della Dichiarazione del 9 maggio 1950. Fu lui, per l'appunto, a disegnare l'as-

${ }_{11}$ V. A. Cohen, Le plan Schuman de Paul Reuter. Entre communauté nationale et fédération européenne, in Revue française de science politique, 1998. 645-663.

12 J. Monnet, Cittadino d'Europa [v. Nota 6], 263. 
setto istituzionale ed a tracciare i poteri dell'Alta autorità della CECA, nonché ad indicarne il nome; fu anche lui l'ispiratore di due frasi centrali della dichiarazione:

L'Alta autorità comune, incaricata del funzionamento dell'intero regime, sarà composta di personalità indipendenti designate su base paritaria dai governi; un presidente sarà scelto di comune accordo dai governi; le sue decisioni saranno esecutive in Francia, Germania e negli altri paesi aderenti. Disposizioni appropriate assicureranno i necessari mezzi di ricorso contro le decisioni dell'Alta Autorità.

A seguito della richiesta di Monnet, Reuter riunì, durante l'estate 1950, il comitato di giuristi che accompagnò la conferenza intergovernativa (Cig) incaricata del negoziato sul futuro Trattato CECA e che partecipò anche attivamente alle discussioni sul futuro Trattato CED. Il libro sulla CECA pubblicato da Reuter nel $1954^{13}$ rimane la migliore guida alla sostanza della politica del mercato del carbone e dell'acciaio come ideato dal trattato e messa in opera nei primi mesi della sua applicazione, a cui è dedicata più della metà del libro (seconda parte) e al sistema istituzionale, a cui è dedicata la prima parte. Accanto a Reuter, l'economista Pierre Uri ebbe anche un ruolo determinante nel concepire il progetto che fu poi presentato ai colleghi d'oltre Reno dell'entourage del Canceliere tedesco Konrad Adenauer, tra cui il giurista Walter Hallstein, che diventò poi il primo Presidente della Commissione CEE nel 1958. La delegazione francese alla Conferneza intergovernativa inaugurata il 3 giugno 1950, che procedde ai negoziati che condussero alla stesura del trattato di Parigi del 18 aprile 1851, era condotta da Monnet e quella tedesca da Hallstein; l'unico uomo politico del gruppo era il deputato italiano Paolo Emilio Taviani, storico e demografo, che era stato segretario della Democrazia Cristiana.

Il metodo comunitario ideato da Monnet, Uri e Reuter e approvato dalle loro controparti nel governo francese e nelle delegazioni alla Cig di Parigi, può essere riassunto in pochi elementi. In primis, iniziare

13 P. Reuter, La Communauté européenne du charbon et de l'acier, Préface de Robert Schuman, Paris, L.G.D.J., 1953. Vale la pena leggere anche il resoconto dall'opera scritto da un altro ordinario di diritto pubblico, Georges Vedel, che è poi stato uno dei componenti importanti della delegazione francese che partecipò alla stesura dei trattati di Roma in «Revue française de science politique», 1954. 899-901. 
con i problemi concreti che richiedono una cooperazione rinforzata tra paesi europei, praticando una politica dei «piccoli passi» che porti a poco a poco a rinforzare i legami tra operatori economici, lavoratori e cittadini di questi paesi. Come secondo elemento, l'affidamento ad un organo indipendente dal governo del compito di identificare soluzione pratiche ai problemi comuni, per superare la naturale tendenza dei governi a vedere solo l'interesse del loro paese piuttosto che non l'interesse comune. Come terzo elemento la rinuncia al diritto di veto come arma della sovranità, che aveva condotto a tante impasse nel periodo tra le due guerre mondiali, anzitutto nel quadro della Società delle Nazioni, di cui Monnet fu Segretario generale dal 1919 al 1923. Egli ricorda così la Società delle Nazioni:

Tra le altre, un'immagine mi è rimasta impressa nella memoria: quella di una seduta del Consiglio in cui si discuteva la ripartizione delle materie prime nel mondo. Il rappresentante dell'Italia, marchese Imperiale, insisteva perché venisse presa una certa decisione. Come al solito lord Balfour, il rappresentante inglese, pareva addormentato. Quando venne il suo turno si alzò e disse semplicemente: "Il governo di Sua Maestà è contro". Poi riprese a dormicchiare. La questione era sistemata. ${ }^{14}$

Ciò significa che in una riunione internazionale, nella quale ogni Stato ha un diritto di veto per via del principio di sovranità, i rappresentanti non hanno bisogno di argomentare la loro posizione. Viceversa, quando si decide a maggioranza, la necessità di cercare alleanze spinge a sviluppare un'argomentazione e quindi a negoziare la propria posizione. Monnet, invece, aveva avuto delle esperienze precedenti in organismi originali segnati da procedure pragmatiche senza uso del veto, come avveniva nella Commissione interalleata in cui si coordinava la logistica a sostegno degli eserciti francese ed inglese durante la Prima guerra mondiale. La comparazione fra queste esperienze spinse quindi Monnet alla convinzione che il diritto di veto era l'impedimento maggiore ad una vera ed efficace cooperazione internazionale. Come quarto elemento del metodo comunitario, vi è l'uso di un sistema di voto ponderato destinato ad impedire sia il dominio dei paesi economi-

14 J. Monnet, Cittadino d'Europa [v. Nota 6], 87. 
camente e demograficamente più forti sia il blocco da parte di un'alleanza di circostanza dei paesi più piccoli. Mi pare utile aggiungere che faceva già parte del metodo comunitario sin dall'inizio un sistema di controllo giurisdizionale delle istituzioni e il loro controllo da una assemblea di tipo parlamentare.

Il metodo comunitario è spesso stato criticato, e lo è ancora oggi, per la sua presunta mancanza di ambizione e per la sua deriva burocratica. Eppure, le proposte più ambiziose di salto verso il federalismo attraverso un atto costituzionale hanno riscontrato una forte resistenza anche quando vi era un progetto firmato all'unanimità dai governi, come nel caso della CED. E utile a questo proposito citare le parole indirizzate da Monnet ai suoi colleghi dell'Alta autorità CECA che presiedeva, il 9 novembre 1954:

I nostri paesi sono diventati troppo piccoli per il mondo attuale, in rapporto ai mezzi tecnici moderni, in confronto all'America e alla Russia. L'unità dei popoli europei negli Stati Uniti d'Europa è il mezzo per rialzare il loro livello di vita e mantenere la pace. Essa è la grande speranza e la grande chance della nostra epoca. Se vi lavoriamo senza indugio e senza tregua, essa è la realtà di domani. ${ }^{15}$

\subsection{L'eredità della CECA nelle istituzioni dell'Unione europea}

Benché, mi pare, sia sempre più spesso dimenticato, è con il trattato CECA e la prassi dei primi anni della Comunità che sono state costruite le fondamenta e l'inquadratura del sistema istituzionale dell'Unione europea.

L'elenco odierno di tali istituzioni, come stabilito all'art. 13 par. 1 del TUE comprende come ben noto «il Parlamento europeo, il Consiglio europeo, il Consiglio, la Commissione europea (in appresso «Commissione»), la Corte di giustizia dell'Unione europea, la Banca centrale europea, la Corte dei conti». Solo le due ultime istituzioni non hanno le loro radici nel sistema CECA: la BCE, istituita nel 1999 sulla base del trattato di Maastricht firmato nel 1992, e la Corte dei conti, istituita nel 1977 sulla base del trattato di Bruxelles del 22 luglio 1975 - che assicurava al Parlamento europeo il diritto di respingere il bilan-

15 J. Monnet, Cittadino d'Europa [v. Nota 6], 355. 
cio e di concedere alla Commissione il discarico, cioè di dare a quest'ultimo atto della corretta esecuzione del bilancio. Tenendo conto che già il trattato CECA dava all'Assemblea parlamentare il potere di adottare una mozione di sfiducia avverso l'Alta Autorità al momento della presentazione della relazione annuale sull'esecuzione del bilancio, anche il trattato di Bruxelles trova in qualche modo le sue radici nel sistema CECA, mentre la BCE è davvero una innovazione istituzionale di grandissima importanza, la prima istituzione di natura veramente federale dell'Unione.

Il Parlamento europeo invece, non è altro che la continuazione dell'Assemblea parlamentare della CECA. Certo, tale Assemblea era composto da membri dei parlamenti degli Stati membri, come dopo, per l'appunto, nei trattati di Roma. Tuttavia, già nel 1954 l'Assemblea approvò la famosa Relazione di Pierre-Henri Teitgen ${ }^{16}$ sui «poteri di controllo dell'Assemblea comune e il loro esercizio» che auspicava l'elezione al suffragio diretto dei suoi membri.

Il Consiglio europeo è stato istituito ben dopo dalla prassi delle riunioni dei capi di Stato e di Governo, a partire dal vertice dell'Aia di dicembre 1969. E tuttavia utile ricordare che durante la prima riunione del Consiglio speciale dei ministri CECA nel 1952, fu il Cancelliere Adenauer, capo del governo federale tedesco, a rappresentare la Germania - anche se lo fece a titolo di Ministro degli esteri, poiché cumulavo tale portafoglio con il carico di Cancelliere. È utile a questo punto ricordare che è stato lo stesso Monnet, ideatore del metodo comunitario a spingere in seguito verso l'istituzionalizzazione dei vertici, con il Comitato d'Azione per gli Stati Uniti d'Europa da lui fondato e presieduto. Lo fece a partire dal vertice del 1972 fino a quello di Parigi del 9-10 dicembre 1974 durante il quale fu deciso, ad iniziativa del Neopresidente della Repubblica francese Valéry Giscard d'Estaing, di far eleggere direttamente il Parlamento europeo a suffragio universale e di riunire almeno due volte l'anno un vertice dei Capi di Stato e di

16 Pierre-Henri Teitgen (1908-1997) era Professore ordinario di diritto pubblico alla Facoltà di giurisprudenza di Parigi uno dei membri fondatori del partito democristiano francese MRP; e stato più volte ministro durante la $4^{\circ}$ Repubblica. E stato uno dei redattori della CEDU e giudice alla Corte EDU. L'edizione del 1977 del suo manuale di diritto comunitario, un'opera magna del ancora molto utile oggi, è stata ristampata di recente: P.-H. Teitgen, Droit institutionnel communautaire, Bruxelles, Bruylant, 2019, 410 p. 
Governo, chiamati per l'appunto «Consiglio europeo». Scrisse Monnet a questo proposito:

Ora le istituzioni europee si erano assunte la responsabilità di immensi settori di attività, sui quali esercitavano la parte di sovranità che era loro delegata. Ma avevano bisogno, per funzionare efficacemente, che i Governi avessero la stessa volontà europea e che, insieme, presentandosi come un'autorità comune, fossero pronti a trasferire l'ulteriore sovranità di cui aveva bisogno una unione europea autentica. La creazione del Consiglio europeo forniva lo strumento di questa indispensabile decisione. ${ }^{17}$

Risuona come un'eco di quest'analisi la decisione presa dalla Convenzione europea del 2002-2003 di lasciare aperta la possibilità di cumulo tra le funzioni di Presidente della Commissione europea e quelle di Presidente del Consiglio europeo.

È vero tuttavia che nel trattato CECA non solo non era previsto il Consiglio europeo, ma «solo» un «Consiglio speciale dei Ministri» che, per di più era concepito piuttosto come un organo tramite il quale $\mathrm{i}$ governi avrebbero dovuto dare il nulla osta alle decisioni proposte dall'Alta autorità. Fu Adenauer, durante la prima riunione del Consiglio speciale dei ministri, a definirne il suo ruolo, come rammentato da Monnet:

Adenauer espose il suo modo di intendere il ruolo del Consiglio, e credo non lo si sia mai definito tanto bene quanto in questo momento iniziale: "Il Consiglio è posto al punto d'incrocio di due sovranità, una sovranazionale e l'altra nazionale... Ma, anche se deve salvaguardare gli interessi nazionali degli Stati membri, esso dovrà stare attento a non considerare questo compito come prioritario. Il suo compito prioritario consisterà piuttosto nel promuovere gli interessi della Comunità, altrimenti quest'ultima non potrà svilupparsi. Per questo lascerà in larga misura all'organismo sovranazionale della Comunità - l'Alta Autorità - la libertà di svilupparsi, e in determinate circostanze dovrà creare questa libertà". ${ }^{18}$

${ }_{17}$ J. Monnet, Cittadino d'Europa [v. Nota 6], 452.

18 J. Monnet, Cittadino d'Europa [v. Nota 6], 339. 
Il Consiglio speciale dei ministri come stabilito dal trattato di Parigi aveva già tutte le caratteristiche dell'odierno Consiglio dell'Unione, composto da membri dei governi degli Stati membri con una presidenza di turno semestrale. L'art. 28 del trattato disponeva come regola di voto un sistema di maggioranza qualificata basato sulla maggioranza assoluta degli Stati membri, includendo almeno uno Stato produttore del $20 \%$ del valore totale della produzione di carbone e d'acciaio della Comunità e, in caso di uguale quota di voti, di quelli di almeno due Stati produttori ciascuno del $20 \%$. Monnet spiega molto bene la ratio dei meccanismi di maggioranza qualificata che fanno parte del metodo comunitario.

Il potere di dire di no costituiva la sicurezza dei grandi nei loro rapporti reciproci e la sicurezza dei piccoli contro i grandi. Ora essi avevano un'ultima occasione di farne un uso assoluto al momento della firma del trattato. Poi, si sarebbe entrati in un mondo sconosciuto, nel quale la maggioranza sarebbe stata la regola, il veto l'eccezione. Ma quale maggioranza? Quattro paesi, quelli del Benelux e l'Italia, rappresentavano non più di un quarto della produzione di carbone e di acciaio. Non sarebbe stato normale che potessero tenere in scacco la Francia e la Germania, cosa che sarebbe accaduta se ciascuno avesse disposto di un voto. Per questa ragione proponemmo un sistema di "voto ponderato" che garantiva che nessuna decisione potesse essere imposta da una coalizione formata solo dalla Francia e dalla Germania, né da quella degli altri riuniti. ${ }^{19}$

Il concetto del voto a maggioranza qualificata in Consiglio è sempre rimasto questo. Non era, invece, previsto l'esistenza del Coreper: l'art. 28 disponeva che il Consiglio comunica con i Stati membri via suo Presidente.

Non solo il Consiglio, ma anzitutto la Commissione, è stata ideata con la Dichiarazione Schuman e il trattato CECA. L'Alta Autorità della CECA è stata concepita come un organo indipendente dai Governi. L'idea di rappresentanza degli Stati membri era però presente nella bozza redatta da Reuter nel 1950 per un accordo franco-tedesco, come ricordato da Monnet:

19 J. Monnet, Cittadino d'Europa [v. Nota 6], 317. 
I principi e gli impegni essenziali qui sopra definiti saranno oggetto di un trattato tra i due Stati. ... L'Autorità incaricata del funzionamento di tutto il regime sarà composta sulla base di una rappresentanza paritetica franco-tedesca e presieduta da una personalità gradita a tutte e due le parti. ${ }^{20}$

Poco dopo, nel progetto preparato per il Governo francese, l'indipendenza della futura Alta Autorità diventava centrale, come sottolineato dallo stesso Monnet:

Fin dal 12 giugno eravamo in grado di presentare a un Consiglio interministeriale francese un progetto che regolava l'indipendenza dell'Alta Autorità e precisava i modi di ricorso contro le sue decisioni. Già si profilavano l'idea di un tribunale arbitrale e la nozione di responsabilità politica dell'esecutivo davanti a un corpo parlamentare. La mozione di censura era esplicitamente prevista. ${ }^{21}$

I due caratteri essenziali della Commissione europea, nonché dell'Alta Autorità CECA, sono l'indipendenza rispetto ai Governi e l'esistenza di potere decisionale incisivo. Un'altra citazione di Monnet è molto utile per comprendere bene di cosa si tratta. Egli scrisse nelle sue Memorie a proposito del progetto di Alta Autorità:

Il lunedì il progetto divenne più strutturato e il sistema istituzionale si rinforzò: l'Autorità internazionale divenne l'Alta Autorità comune. Nella quarta versione essa è qualificata come sovranazionale, ma questa parola non mi piaceva e non mi è mai piaciuta. Ciò che importava era la funzione che essa implicava e che si trovava meglio espressa nella versione seguente con questa frase: "le decisioni dell'Alta Autorità sono esecutive in Francia e in Germania, e negli altri paesi aderenti”. Un tale potere esigeva garanzie, e il principio dei ricorsi sarà introdotto senza maggiori precisazioni. ${ }^{22}$

Tali maggiori precisazioni furono, invece, introdotte nella bozza di trattato preparata dal gruppo di collaboratori di Monnet nei primi tre mesi del 1951, bozza della quale egli ricorda così:

20 J. Monnet, Cittadino d'Europa [v. Nota 6], 265.

21 J. Monnet, Cittadino d'Europa [v. Nota 6], 288.

22 J. Monnet, Cittadino d'Europa [v. Nota 6], 266. 
cette rédaction était pour l'essentiel l'œuvre conjointe d'Uri et d'un grand juriste, Maurice Lagrange. La chance nous avait encore servis lorsqu'au début de l'automne, j'avais demandé à Parodi de nous désigner un membre du Conseil d'Etat pour veiller à la rigueur des textes conçus pour durer cinquante ans et servir de modèle à des futurs traités européens. Le sort tomba sur un austère et modeste conseiller du contentieux qui se vit projeté directement de ses grimoires dans le monde effervescent de la rue de Martignac. Je revois l'homme grand et droit, au visage anguleux et pâle, de la race des magistrats de parlement qui tinrent anonymement pendant des siècles la France en état. In entre dans mon bureau et je lui dis: "Monsieur Lagrange, vous allez rédiger le traité". Calmement, il me répond: "Je ne sais pas de quoi il s'agit, mais je m'efforcerai de le faire bien, Monsieur le Président”. L'instant, d'après, il était au travail et sa contribution à l'œuvre fut inestimable. ${ }^{23}$

Ricordi come quelli di Monnet, nonché la stesura dell'art. 33 (dedicato al ricorso in annullamento) del Trattato CECA hanno fondato l'idea secondo la quale il sistema del contenzioso comunitario altro non fosse che una mera trasposizione delle regole del contenzioso amministrativo francese. Invece, un'analisi più precisa delle disposizioni vigenti - quasi immutate sin dai Trattati di Roma - mette in luce quanto anche il contributo dei giuristi italiani e tedeschi sia stato importante, il che ha portato ad un sistema di ricorsi specifico, diverso da quello abitualmente conosciuto negli Stati membri. È da precisare che il trattato CECA non prevedeva la figura degli avvocati generali, che fu tuttavia creata sin dall'inizio del funzionamento della Corte. Maurice Lagrange fu nominato come avvocato generale 4 dicembre 1952 insieme ai primi giudici, seguito qualche mese dopo da Carl Roemer. L'avvocato generale, come noto, è una figura che riproduceva, per l'appunto, quella del commissaire du gouvernement - ora rapporteur public - presso il Consigli di Stato francese. La presenza di Lagrange tra i magistrati della Corte fu particolarmente importante poiché il primo giudice francese, nominato lo stesso giorno, non era un giurista, ma un alto funzionario di formazione economica; si ricorda che fu Lagrange a sviluppare il concetto di principi generali del diritto comuni agli Stati membri nella causa Algera, ${ }^{24}$ e che egli ebbe un

${ }_{23}$ J. Monnet, Mémoires, Paris, Fayard, 1976, 513, non ripreso nella traduzione italiana.

${ }^{24}$ Sentenza 11 luglio 1957, cause riunite 7/56 e da 4/57 a 7/57, Algera. 
ruolo determinante nella formazione della giurisprudenza sul primato, essendo, per l'appunto l'avvocato generale nella causa Costa c. Enel. ${ }^{25}$

Il sistema istituzionale CECA comportava anche un Comitato consultativo, antenato del Comitato economico e sociale del trattato CEE, sia nella sua composizione sia nel suo funzionamento. $\grave{E} \mathrm{da}$ aggiungere che anche le prime agenzie europee sono nate nel sistema CECA, con l'Ufficio comune dei consumatori di rottame e la Cassa di perequazione per il rottame importato, ben noti degli studiosi della giurisprudenza della Corte di Giustizia, poiché la Meroni ${ }^{26}$ riguardava, per l'appunto, la legittimità dell'istituzione di questi due organismi non previsti dai trattati, nonché i limiti delle possibilità di delega di potere effettuati dall'Alta Autorità a tali organismi. La giurisprudenza Meroni rimane il riferimento imprescindibile della disciplina della delega in diritto dell'Unione europea.

Da menzionare come eredità istituzionale è anche il regime linguistico dell'Unione. Benché il trattato CECA fosse stato redatto in lingua francese e solo la versione francese facesse fede, fu concluso il 24 luglio 1952 - un giorno dopo l'entrata in vigore del trattato CECA - un accordo intergovernativo che è senza dubbio ${ }^{27}$ all'origine del regolamento $1 / 58$

25 Causa 6/64.

26 Sentenza 13 giugno 1958, causa 10/56, Meroni.

${ }^{27}$ V. Il testo, non facile da rintracciare poiché non è mai stato pubblicato in via ufficiale, è riprodotto in P. Reuter, La Communauté européenne du charbon et de l'acier, Préface de Robert Schuman, Paris, L.G.D.J., 1953, 81: «1. Les langues officielles et les langues de travail de la Communauté sont : français, allemand, italien et néerlandais; 2. Les décisions, recommandations et avis individuels sont rédigés dans la langue de ceux qu'ils concernent ; il en est de même de la correspondance adressée aux entreprises; 3 . La correspondance adressée aux institutions de la Communauté est adressée, au choix du correspondant, dans l'une des langues officielles; la réponse est adressée dans la même langue; 4. L'Assemblée règle elle-même, en ce qui la concerne, les questions pratiques relatives à l'usage des langues, les délégués pouvant s'exprimer dans l'une quelconque des langues officielles; 5. La langue parlée et écrite en usage devant la Cour est déterminée comme suit : a) dans les litiges entre la Communauté ou les institutions d'une part, et un Etat membre, une entreprise ou une personne ressortissant de cet Etat membre d'autre part, la langue de procédure est la langue nationale de cet Etat; b) dans les litiges entre Etats membres, la langue de procédure est la langue nationale de la partie défenderesse; c) la langue de procédure s'entend notamment de la langue des requêtes, mémoires, défenses, observations, documents, procès-verbaux, plaidoiries et des arrêts et toutes autres décisions de la Cour. La langue dans laquelle est rédigé le projet d'arrêt est déterminée par la Cour ; si cette langue n'est pas celle de la procédure, 
sul regime linguistico delle Comunità, ${ }^{28}$ adottato su base del trattato di Roma. Vi era stabilito il principio della parità linguistica per lingue ufficiali e lingue di lavoro - nel caso di specie francese, italiano, olandese e tedesco; oggigiorno le 24 lingue dei trattati $-{ }^{29}{ }^{29}$ il diritto di rivolgersi a e di ricevere risposta dell'Alta autorità nella lingua scelta da chi faceva la domanda, nonché la facoltà per gli Stati membri in cui vi era più di una lingua ufficiale (all'epoca il Belgio e il Lussemburgo) di indicare quali lingue potevano essere utilizzate a tale scopo. L'accordo stabiliva anche il regime linguistico della Corte, poi ripresa nello Statuto.

Di fondamentale importanza come eredità del trattato CECA è anche il concetto di trasparenza, pur essendo troppo spesso attribuito solo all'influenza degli Stati nordici - Danimarca sin dal 1973, Svezia e Finlandia sin dal 1995. Ė vero che il trattato CECA non usava il termine di "trasparenza" ma quello di "pubblicità". L'art. 15 del trattato, per l'appunto; è la prima norma in Europa ad aver stabilito un obbligo generale di motivazione ${ }^{30}$ di tutte le decisioni, ben prima di Stati membri come la Germania nel 1975, la Francia nel 1979 o l'Italia nel 1990, e solo cinque anni dopo gli Stati Uniti d'America con l'Administrative Procedure Act federale del 1946. ${ }^{31}$ L'art. 47 prevedeva

le texte de l'arrêt est ensuite établi dans la langue de procédure sous la responsabilité de la Cour ;d) si les parties au litige sont d'accord sur l'emploi d'une autre langue officielle, la Cour peut autoriser l'emploi de cette langue comme langue de procédure; 6 . Les règlements, décisions générales et autres délibérations générales des institutions de la Communauté sont publiées dans le Journal Officiel de la Communauté dans les quatre langues officielles. 7. En ce qui concerne les Etats membres où en vertu de la Constitution, existent plusieurs langues officielles, l'usage de la langue sera, à la demande de l'Etat intéressé, déterminé selon les règles générales découlant de la législation de cet Etat". Per maggiori precisazioni, v. J. Ziller, Le multilinguisme, caractère fondamental du droit de l'Union européenne, in M. Condinanzi, V. Canizzarro, R. Adam et al. (a cura di) Liber amicorum Antonio Tizzano. De la Cour CECA à la Cour de l'Union: le long parcours de la justice européenne, Torino, Giappichelli, 2018, 1067 ss.

${ }_{28}$ Regolamento $\mathrm{n}^{\circ} 1$ che stabilisce il regime linguistico della Comunità Economica Europea, GUCE 17 del 6.10.1958, pag. 385.

29 La Brexit non cambia niente alla sostanza del regime linguistico dell'Unione, poiché l'inglese è lingua ufficiale dell'Irlanda e di Malta secondo le loro costituzioni nonché nella prassi giuridica; v. J. Ziller, Le multilinguisme, caractère fondamental du droit de l'Union européenne [v. Nota 27] 1079.

30 La legge austriaca sul procedimento del 1925 prevedeva un obbligo assoluto di motivazione solo per le decisioni di seconda istanza delle autorità amministrative.

${ }^{31}$ Per maggiori dettagli v. J. Ziller, Origines et retombées du principe de 
anche l'obbligo per l'Alta Autorità di pubblicare i dati che avrebbero potuto essere utili ai governi ed a tutti gli altri interessati. Il principio di pubblicità era anche centrale nel sistema del mercato comune del carbone e dell'acciaio in quanto disciplina di promozione e controllo della concorrenza.

\subsection{L'eredità della CED e della CPE nelle istituzioni dell'Unione europea}

Oltre alle istituzioni, principi e regole specifiche alla materia della CED e a quelle della CPE, vi sono elementi non ancora sviluppati nel sistema CECA che meritano secondo me di essere ricordati.

Un primo elemento da mettere in luce come antenato delle discipline istituzionali attuali è quello della redazione del progetto di statuto della CPE. Il sistema della Convenzione attualmente previsto all'art. 48 TUE ha, benché ripreso dall'esperienza del 2000 per la stesura della Carta dei diritti fondamentali e da quella della Convenzione europea 2002-2003, un precedente lontano. Il 30 maggio 1952, tre giorni dopo la firma del Trattato CED - e quasi due mesi prima dell'entrata in vigore del trattato CECA - l'Assemblea consultiva del Consiglio d'Europa chiese ai governi dei sei Stati fondatori di affidare l'Assemblea della CECA - che doveva diventare l'Assemblea del CED secondo il nuovo trattato - la stesura di uno statuto politico europeo che consentisse il controllo democratico del futuro esercito europeo. L'Assemblea CECA, aumentata da altri tre delegati francesi, tre italiani e tre tedeschi, nominò, quindi una Commissione costituzionale per redigere il progetto. Si può anche ricordare che, quando l'Assemblea parlamentare delle Comunità decise di cambiare il suo nome in Parlamento europeo nel 1962, vi furono delle critiche in Francia da parte di alcuni politici gollisti riguardo alla presunta volontà di trasformarlo in organo costituente. Invece è solo dopo la prima elezione diretta del Parlamento europeo nel 1979 che inizio il lavoro che portò all'adozione della risoluzione che approvò la proposta di Trattato d'Unione europea di cui A. Spinelli era relatore. Il precedente dell'Assemblea ad-hoc era di sicuro presente nella mente dei sostenitori del referendum di indirizzo del 18 giugno 1989 svoltosi in Italia con il quesito:

transparence en droit de l'Union europénne, in G.J. Guglielmi, E. Zoller (dir.), Transparence, démocratie et gouvernance, Paris, Editions Panthéon Assas, 2014, 25-45. 
Ritenete voi che si debba procedere alla trasformazione delle Comunità europee in una effettiva Unione dotata di un governo responsabile di fronte al Parlamento, affidando allo stesso Parlamento europeo il mandato di redigere un progetto di costituzione da sottoporre direttamente alla ratifica degli organi competenti degli Stati membri della Comunità? ${ }^{32}$

Uno degli elementi maggiori nel contesto dell'epoca che dimostra che il trattato CECA non creava un embrione di federazione europea, è che si applicava solo al territorio europeo dei suoi Stati membri in virtù del suo articolo 79. L'estensione degli imperi coloniali all'epoca e l'estrema diversità delle formule giuridiche che collegavano territori non europei alle loro madrepatrie spiegano la loro esclusione dall'ambito di applicazione del trattato CECA. Essi non costituivano un mercato unico con le loro rispettive madrepatrie. Inoltre, il trattato è stato progettato per le particolari strutture dell'industria siderurgica e dell'industria carbonifera nell'Europa occidentale e in particolare per il bacino della Ruhr e della Lorena, in Lussemburgo. L'art. 79, lettera d, frase 2), prevedeva solo che "ciascuna Parte contraente si impegna ad estendere agli altri Stati membri le misure preferenziali cui essa gode per carbone e acciaio nei territori non europei soggetti alla sua giurisdizione".

Questa ultima clausola non era altro che una logica applicazione della clausola della nazione più favorita ben nota dal diritto internazionale pubblico, e aveva il solo scopo di evitare discriminazioni tra gli Stati membri nel settore del commercio dei prodotti contemplati dal trattato che sarebbe potuto derivare dai rapporti privilegiati tra le madrepatrie e le loro colonie o territori d'oltremare. Tuttavia, il governo italiano avrebbe gradito l'estensione del trattato CECA al Nord Africa, al fine di garantire la fornitura di minerale di ferro alle acciaierie nel sud della penisola. Infatti, un Protocollo sul regime da applicare ai prodotti della CECA nei confronti dell'Algeria e dei dipartimenti d'oltremare della Repubblica francese è stato poi allegato ai trattati di Roma. Esso stabiliva che gli Stati membri risolvessero il problema in uno spirito di reciproca collaborazione quanto prima, al più tardi in

32 Sul referendum stesso, v. l'editoriale del «Il Federalista», Il primo referendum per la Costituente europea, 1989, 3 ss. 
occasione della prima revisione del trattato che istituisce la CECA. Quando ebbe luogo questa prima revisione - con il Trattato di fusione del 1965 - non solo il Marocco e la Tunisia, ma anche l'Algeria, erano indipendenti.

Poiché viene sempre più sovente dimenticato, è utile ricordare che tra i paesi fondatori delle Comunità solo il Lussemburgo non aveva mai avuto un impero coloniale, e che la Germania ne aveva avuto uno fino alla fine della Prima guerra mondiale. È vero che la decolonizzazione era già iniziata per tre dei sei stati fondatori al momento della dichiarazione Schuman. I Paesi Bassi dovettero concedere l'indipendenza all'Indonesia nel 1949 a seguito di un conflitto armato di due anni. I mandati delle Nazione Unite concessi alla Francia su Libano e Siria erano terminati nel 1946 e quello dell'Italia su Tripolitania e Cirenaica (attuale Libia) il 24 dicembre 1951. Ma l'era degli imperi coloniali non era finita, né per gli Stati fondatori della CECA, né per il Regno Unito - benché l'India fosse indipendente sin dal 15 agosto 1947 -, né per la Spagna e il Portogallo, che aderiranno alle Comunità più tardi. In Africa, il Belgio aveva le colonie del Congo e del Ruanda-Urundi. La Francia era presente con i dipartimenti dell'Algeria, con i territori d'oltremare dell'Africa Equatoriale e dell'Africa Orientale Francesi, così come quello di Gibuti, esercitava il mandato di tutela concesso dalle Nazione Unite su Togo e Camerun (per quest'ultimo insieme a Regno Unito), ed esercitava protettorati sul il Marocco e la Tunisia. L'Italia esercitava il mandato di tutela concesso dalle Nazione Unite sulla Somalia. Per quanto riguarda gli Stati che hanno aderito più tardi, le colonie spagnole e portoghesi in Africa erano ancora intatte, così come quelle del Regno Unito, che tuttavia aveva abbandonato ogni responsabilità per l'Egitto nel 1953.

In America, Francia e Paesi Bassi erano ancora presenti nel continente sudamericano (Guiana francese e olandese) e nelle Antille (Guadalupa e Martinica, Curaçao e dipendenze), cosi come la Gran Bretagna (Guyana e British West Indies, a cui si aggiungeva il Belize in America centrale). Per quanto riguarda il Nord Atlantico, St. Pierre e Miquelon vicino alla costa canadese sono francesi, le Bermude sono britanniche, le Azzorre e Madera portoghesi, le isole Canarie spagnole. Nel Pacifico la Francia era presente in Nuova Caledonia, negli stabilimenti francesi dell'Oceania (oggi Polinesia Francese), nel Protettorato di Wallis e Futuna, e nel condominio franco-britannico delle Nuove Ebridi (ora Vanuatu); i Paesi Bassi erano ancora presenti nella Nuova 
Guinea olandese e il Portogallo a Timor (sebbene la sua sovranità non sia più effettiva dall'indipendenza dell'Indonesia); il Regno Unito, oltre al suo condominio con la Francia, aveva anch'essa molti territori e colonie.

Nell'Oceano Indiano la Francia era ancora presente in Madagascar, nelle Comore, alla Réunion, il Regno Unito alle Maldive, a Mauritius e nell'arcipelago di Chagos. L'indipendenza dell'India era, certo, un dato di fatto sin dalla Dichiarazione di Attlee del 20 febbraio 1947 rispetto ai territori sovrani del Regno Unito; ma gli insediamenti francesi di Chandernagor, Karikal, Mahé, Pondicherry e Yanaon furono ceduti al governo indiano solo il 21 ottobre 1954 e quello portoghese di Goa fu annesso dall'India solo nel dicembre 1961. Infine, in Estremo Oriente, la Francia era ancora presente in Cambogia, Laos e Vietnam, benché la guerra d'indipendenza d'Indocina fosse già iniziata nel 1945 quando i territori erano sotto controllo giapponese. Il Regno Unito era presente in Birmania e in Malesia. Hong Kong era ancora una colonia britannica e Macao una colonia portoghese.

Il progetto di Comunità europea di difesa non si applicava neanche ai territori d'oltremare dei suoi Stati membri, ad eccezione dell'Algeria e di Saint Pierre e Miquelon. Questa eccezione è il risultato degli stretti legami tra la CED e il trattato del Nord Atlantico.

I primi due trattati comunitari sono stati contrassegnati dalla loro natura funzionale, che spiega il modo in cui trattano i territori d'oltremare. Con l'aggiunta di una più ampia dimensione politica e la presa in conto dei cittadini nell'integrazione europea nel progetto di CPE l'approccio cambia. Il parlamento bicamerale previsto dallo Statuto doveva includere una Camera dei Popoli eletta direttamente e un Senato nominato dai parlamenti nazionali. Insieme avevano il potere di fare leggi europee. I popoli d'oltremare erano debitamente presi in considerazione dall'articolo 15 del progetto: benché lo statuto prevedesse un numero uguale di deputati eletti nei territori di Germania, Francia e Italia, era aggiunto che "Tuttavia, una rappresentanza aggiuntiva è riconosciuta alla Repubblica francese in relazione ai suoi dipartimenti e territori d'oltremare, alle condizioni stabilite da una legge francese".

Quindi il paragrafo 3 prevedeva dell'art. 15 attribuiva 70 deputati alla Francia, 63 alla Germania e all'Italia, 30 al Belgio e 30 ai Paesi Bassi, e infine 12 al Lussemburgo. Il progetto di Statuto prevedeva un sistema parlamentare bicamerale simile più a quello degli Stati Uniti d'America che al sistema della Repubblica federale di Germania a cui, 
invece, assomigliava il sistema CECA e quello odierno dell'Unione, dove il Consiglio dell'Unione si può paragonare al Consiglio federale (Bundesrat).

Lo statuto della Comunità politica europea avrebbe dovuto applicarsi (articolo 101, paragrafo 1) "a tutti i territori soggetti alla giurisdizione di ciascuno Stato [...] a meno di una dichiarazione fatta prima della firma del trattato da parte di uno Stato membro interessato".

D'altra parte, è stato previsto (articolo 101, paragrafo 2) che "le leggi, le raccomandazioni e le altre decisioni della Comunità, nonché i trattati da essa conclusi, si applicano ai territori non europei solo alle condizioni di adattamento determinate dallo Stato membro a cui appartengono".

L'articolo 90 prevedeva la possibilità per la Comunità di concludere trattati o accordi di associazione con uno Stato non membro europeo o con uno Stato d'oltremare collegato ad uno Stato membro per via di disposizioni costituzionali.

Colpisce quindi la presenza nel progetto di trattato CPE di un Parlamento bicamerale da paragonare a quello degli Stati Uniti d'America. Inoltre, è da sottolineare che il sistema odierno degli artt. 355 TFUE rispetto al campo di applicazione territoriale è basato su concetti e regole già presenti, per l'appunto nel progetto CPE.3.

\subsection{L'eredità della CECA e la Brexit}

Jean Monnet è sempre stato del parere che la partecipazione del Regno Unito nell'unione dell'Europa fosse indispensabile. Nelle sue Memorie egli riferisce sulle discussioni che ebbe con i Britannici nei primi di giugno del 1950, quando egli fece loro la proposta di partecipare alla futura Alta Autorità del carbone e dell'acciaio.

Nei giornali, alla Camera dei Comuni e in seno ai partiti si sarebbe impegnato un appassionato dibattito. E tale dibattito sintetizzava già da allora gli stati d'animo che l'Inghilterra avrebbe sperimentato per

33 Per maggiori precisazioni v. J. Ziller, La tutela dei diritti tra campo di applicazione personale e campo di applicazione territoriale del diritto dell'Unione europea, in U. Leanza, A. Tizzano et al., Scritti in onore di Giuseppe Tesauro, Editoriale Scientifica, Napoli, 2014, Vol. I, 749 s. 
venticinque anni di fronte al problema dell'Europa, fino al referendum popolare che nel 1975 mise fine alle sue esitazioni. Vi si ritrova allo stato primitivo, libera da quelle scorie che la polemica sui costi economici del Mercato comune vi avrebbe introdotto più tardi, l'immagine degli inglesi sul loro destino nazionale. ${ }^{34}$

Citando un documento di un vertice del partito laburista, pubblicato il 13 giugno 1950, Monnet continua così:

Vi si affermava il rifiuto di leggi comuni, e in particolare della regola della maggioranza nelle relazioni tra i popoli, e ugualmente, vi erano chiaramente enumerati due principi fondamentali della politica inglese di allora: nessun cambiamento nei rapporti fra Gran Bretagna e l'Europa occidentale deve intervenire a diminuire la sua posizione di centro determinante del Commonwealth e come banchiere della zona sterling. Nessuna delega di potere può essere consentita ad una autorità sopranazionale che verrebbe interferire nell'esperienza socialista britannica. Infine si poteva leggere questa frase rivelatrice: "Per lingua, origini, costumi, convenzioni politiche e interessi, noi siamo più vicini all'Australia e alla Nuova Zelandia che all'Europa". ${ }^{35}$

Sembra che, con qualche aggiornamento, l'analisi fosse ancora valida sessant'anni anni dopo, al momento del referendum del 23 giugno 2016, con una non piccola differenza: nel 1976, quando scrisse le sue Memorie, Monnet, come tanti altri, pensava che il referendum positivo dell'anno precedente sul mantenimento del Regno Unito nelle Comunità avrebbe sancito la fine delle "esitazioni" britanniche verso il progetto d'integrazione dell'Europa. Purtroppo, non fu così.

Secondo le sue Memorie, disse Monnet nel 1959 al ministro dell'economia e padre del "miracolo economico" tedesco del dopoguerra, Ludwig Erhard:

“Nessuno più di me desidera la vedere gli Inglesi nell'Europa, ma non li vedremo, se si faranno loro troppe concessioni all'inizio". ${ }^{36}$ Non era, questo, un punto di vista tattico adatto al momento particolare, ma

34 J. Monnet, Cittadino d'Europa [v. Nota 6], $282 \mathrm{s.}$

35 J. Monnet, Cittadino d'Europa [v. Nota 6], $282 \mathrm{~s}$.

36 J. Monnet, Cittadino d'Europa [v. Nota 6], 394. 
un convincimento costante che mi ero formato nel corso dei miei numerosi negoziati con gli inglesi. Il loro carattere nazionale li induce a ricercare nei loro rapporti con gli altri una posizione privilegiata che li dispensi dal dover cambiare.

Chi scrive è sicuro che Michel Barnier, capo negoziatore dell'Unione europea per l'accordo di recesso, conosceva e condivide la posizione cosi illustrata. Monnet aggiungeva quasi immediatamente "La civiltà occidentale ha bisogno dell'Inghilterra, e l'Europa, per conservare l'insostituibile contributo che essa dà a questa civiltà, ha bisogno delle capacità del popolo inglese”. E scrisse poi:

Tra tutti i progressi che la civiltà ha fatto grazie agli inglesi, due mi sembrano essenziali: il rispetto della libertà e il funzionamento delle istituzioni democratiche. Che cosa sarebbe la nostra società senza l'babeas corpus e senza il sistema parlamentare che fa da contrappeso al potere esecutivo? Non basta che ne abbiano inventato il principio e che noi lo abbiamo imitato, ci resta da viverlo nella pratica quotidiana. E su questo punto, l'Inghilterra e i paesi del continente, esercitando in comune la democrazia, possono scambiarsi molti insegnamenti. Gli inglesi comprendono le istituzioni ed il modo di servirsene meglio dei popoli continentali. Questi pensano che siano gli uomini a regolare le questioni, e certamente gli uomini sono importanti, ma senza le istituzioni non decidono nulla di grande e di duraturo: e questo, gli inglesi lo sanno sin da molto tempo. ${ }^{37}$

Sembra, purtroppo, che la classe politica britannica abbia dimenticato quest'insegnamento negli ultimi anni, dando, invece, il cattivo esempio dell'uso sconsiderato del referendum a fini populisti.

3. CENNi RELATivi Alle politiche E AL DiRitTo MATERIALE DELLA COMUNITÀ EUROPEA DEL CARBONE E DELL'ACCIAIO

Chi scrive, quando spiega il Trattato CECA ai suoi studenti, e per far capire che il progetto di integrazione non è stato pensato dai burocrati per i burocrati, cita sempre la canzone che cantavano i loro nonni,

37 J. Monnet, Cittadino d'Europa [v. Nota 6], 395. 
"Marina" poiché è stato realizzato anche un film sulla vita dell'autore della canzone Rocco Granata, con un regista belga, Peter Bouckaert. Nel film si vede la famiglia del cantautore nelle miniere nel Belgio degli anni 1947-1948 ove gli italiani sono discriminati rispetto i belgi: non hanno ad esempio infatti accesso alle stesse case. Di colpo poi tutto cambia: italiani e belgi hanno gli stessi diritti. Nel film non si comprende bene il motivo di questo cambiamento, ma noi sappiamo che era entrato in vigore il Trattato CECA il quale si occupava anche dei diritti dei lavoratori. Questo è uno degli elementi che viene spesso dimenticato: proprio in Italia il centro di ricerca di Ispra ha iniziato la sua attività di ricerca incentrandosi sul miglioramento della sicurezza dei lavoratori nel settore del carbone e dell'acciaio. Ciò è molto interessante, sia guardando alle disposizioni del Trattato CECA che sono già di per sé precise, sia guardando alle disposizioni di diritto derivato adottato all'epoca dalle autorità della CECA..$^{38}$

Prendendo sempre a riferimento gli aspetti meno conosciuti della CECA, si può anche vedere come vi sia una traccia di politica ambientale ${ }^{39}$ in un'epoca ove non si parlava assolutamente di questi temi: nello specifico non sono utilizzati i termini "ambientale" ma vi sono delle disposizioni nel Trattato e pure della giurisprudenza della Corte di giustizia della CECA che ha sovente insistito sullo "sfruttamento razionale delle risorse naturali”. Tale aspetto era molto importante per l'Italia, dal momento che il settore siderurgico era costituito soprattutto da piccole e medie imprese che lavoravano coi rottami, e conseguentemente, vi era l'idea che il rottame fosse considerato una risorsa utile che permetteva di sfruttare al meglio le risorse naturali. Tale idea è tutt'ora attuale e presente nel dibattito odierno sul cambiamento climatico. Ciò per confermare l'ipotesi per cui nel Trattato CECA vi è già qualcosa di più delle semplici disposizioni sulle istituzioni.

Vale la pena ricordare che vi era un sistema di finanziamento dell'Alta autorità basato su contributi delle imprese del settore che ha funzionato più o meno bene, ma che rimane fonte di ispirazione per

38 V. G. Avanzini, Le origini della tutela dei lavoratori e della tutela dell'ambiente nel Trattato e nel diritto derivato CECA in G. Rossolillo (a cura di), L'integrazione europea prima dei trattati di Roma, 110-116.

V. G. Avanzini, Le origini della tutela dei lavoratori e della tutela dell'ambiente nel Trattato e nel diritto derivato CECA in G. Rossolillo (a cura di), L'integrazione europea prima dei trattati di Roma, 116-119. 
altri organi: ${ }^{40}$ vi sono alcune agenzie dell'UE oggi che vivono ancora di tali contributi.

Due osservazioni a mo' di conclusione sul contenuto delle politiche dell'epoca. Quando si parla della CECA la tentazione è quella di considerare il tema industriale come meno problematico rispetto a tematiche attuali quali l'immigrazione o la moneta unica; chi scrive insiste sul punto sempre anche con gli studenti ricordando come, all'epoca, il settore siderurgico fosse fondamentale per la sovranità. Se è nato il progetto della CECA il motivo risiede nel fatto che, sia dal lato francese e sia tedesco, politici lungimiranti si erano resi conto che la gestione del bacino del carbone e dell'industria dell'acciaio della Ruhr non poteva proseguire senza danni con la supervisione delle autorità di occupazione americane, francese ed inglese che gestivano le risorse e produzione dell'acciaio tramite l'Autorità internazionale della Ruhr. Ciò dimostra bene come, anche se una materia è strettamente connessa al concetto di sovranità, laddove vi siano le condizioni, si può andare verso un sistema più integrato.

Seconda osservazione: Francia e Germania hanno firmato il 22 gennaio 2019 il Trattato di Aquisgrana, ${ }^{41}$ con cui si integra il trattato dell'Eliseo del 22 gennaio $1963 .{ }^{42}$ Le reazioni nella stampa italiana e nella politica italiana son state piuttosto critiche, sottolineando come nuovamente Francia e Germania vorrebbero imporre un direttorio all'Europa. Invece, già nel febbraio 2018 era stato istituito un gruppo di lavoro franco-italiano per la stesura di un "Trattato del Quirinale" che potesse rispecchiare in qualche modo il trattato dell'Eliseo. Infatti, si ricorda che già il progetto CED era un'iniziativa franco-italiana, così come il progetto di uno Statuto della Comunità europea.

A fine 2019 e anche stato pubblicato un documento di riflessione franco-tedesco per un cd. Franco-German non-paper per la preparazione della futura conferenza sul futuro dell'Europa. ${ }^{43} \mathrm{E}$ interessante vede-

40 V. A. Zatti, Le finanze della CECA: spunti e riflessioni sul futuro della UE in G. Rossolillo (a cura di), L'integrazione europea prima dei trattati di Roma, 57-93.

${ }_{41}$ V. https://www.diplomatie.gouv.fr/IMG/pdf/traite.aix-la-chapelle.22.01. 2019_cle8d3c8e.pdf

${ }_{42}$ V. https://www.france-allemagne.fr/Traite-de-l-Elysee-22-janvier-1963.html\#

43 V. https://ec.europa.eu/dorie/fileDownload.do;jsessionid=W6CqVKmoqpc HZhjR9CT8F5k6Ql1xBKRrEFN_B5BGeHPm2ffp0m9d!1094967622?docId=633954 0\&cardId $=6339539$ 
re come questo documento prevede due fasi di riflessioni: la prima sulle istituzioni e i processi democratici nonché il rispetto dello stato di diritto, un tema quasi scontato ai tempi della Dichiarazione Schuman ove era chiaro cosa volesse dire vivere senza stato di diritto, ma di nuovo attuale viste le situazioni di Polonia e Ungheria. L'altra fase di riflessione è invece incentrata sulle politiche da sviluppare. Qui, riflettendo su come rafforzare il mercato interno, vale a dire l'aspetto più solido fino ad ora creato dalle comunità, si potrebbe pensare addirittura a quanto fatto già nel settore siderurgico e procedere in tal senso anche per il mercato interno.

Guglielmo il Muto aveva come motto: Point n'est besoin d'espérer pour entreprendre, ni de réussir pour persévérer - Non c'è necessità di sperare per intraprendere, né di succedere per perseverare... 
